\title{
FPGA Based Optimized Reconfigurable Base-2 Constant Coefficient Multiplier Architecture for Image Filtering
}

\author{
N. Sambamurthy, M. Kamaraju
}

\begin{abstract}
Image convolution using FPGA has been comprehensively used for noise removal of Reconfigurable computing based image Processing Algorithm. Particularly these filters are widely used in embedded computer vision applications like edge detection and Feature extraction analysis. Practical implementation of filter requires enormous computational requirement. The multiplier plays very important role in the image convolution. The existed multiplier design requires more computational complexity for the $3 \times 3$ test image. For this the proposed reconfigurable constant coefficient multiplier uses base-2 Common sub expression algorithm. which reduces the computational complexity in a better way. The proposed 2D-convolution in image application is the value of resultant output is multiplication of image pixel with corresponding kernel value. In this work the realization of $2 D$ convolution to be done using proposed constant coefficient multiplier and analyzed using Xilinx Virtex-5 FPGA platform
\end{abstract}

Keywords: Field Programmable Gate array (FPGAs), Filters, 2D Filters, Gaussian Mask; BCSE;Mask, Image controller;

\section{INTRODUCTION}

In spatial Image convolution [1], the each output image pixel is computed by multiplying the values of each input image pixel with the corresponding kernel value. The convolution tap output gives the resultant pixel output. As shown in Fig.1.

Usually to compute the filtering operation for a given symmetric Gaussian kernel size of $3 \times 3$ and $3 \times 3$ image requires 9 multiplications and 8 summing elements are required.[10]-[11]. The digital convolution [2]-[3] process widely implemented in FPGA [4] for several decades. However these algorithms are computationally expensive.

Revised Manuscript Received on August 20, 2019.

* Correspondence Author

N.Sambamurthy*, E.C.E Dept, Gudlavalleru Engineering College ,Gudlavalleru, India, sambanaga009@gmail.com

Dr. M. Kamaraju, E.C.E Dept, Gudlavalleru Engineering College ,Gudlavalleru, India

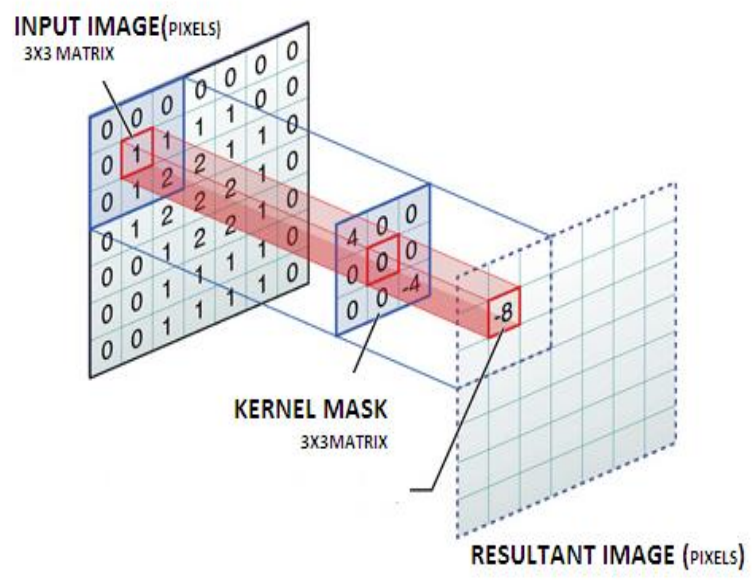

Fig.1.2 Dimensional - Image convolution

\section{Literature SURVEY}

Nowadays, the image Convolution process is implemented on Graphical processing hardware (GPU) and DSP shows inadequate performance and high outlay. Moreover, ASIC's with rigidity and performance is the main drawback [4]-[8]. Generally, to perform a 2D-image convolution with FPGA, used 65,536 product terms and 65,535 adders are required for $256 \times 256$ gray scale image. First, the image size of $3 \times 3$ and a kernel mask size of $3 \times 3$ were chosen for performing the spatial convolution operation[5].

The Multiplier based convolution hardware [1],[2] is needed for noisy images and video processing. In linear spatial filtering, the multiplier is tobe optimized key component and defines the performance of the linear filter hardware [5],[6]. Therefore, reconfigurable fixed coefficient multiplier hardware architecture for spatial filter has been focused continuously [7].

C.Y.YAOet.al., implemented computational efficient multiple coefficient multiplication (MCM) algorithm for image filtering is designed [8].This MCM algorithm [8] mainly used graph algorithm and common sub-expression algorithm used. The image filtering does pixel by pixel multiplication using the MCM design uses more area and high complexity.

For portable devices The MCM design is not suitable. F. Boracchi introduced for optimal complexity design based multiplier for low power applications. But the multiplier design is generic 
and not suitable for filtering [3]. A.G.Dimster suggested the Fixed binary common sub expression algorithm (FBCSE) is eliminate the computational complexity moderately by considering BCSE algorithm across the adjacent coefficients is designed [4],[9],[10].

The Fixed bit common sub expression algorithm [1],[9] Challenges the problem with redundant computational complexity.

The algorithms on a fixed coefficient design are not applicable for Image filter. Because the kernel coefficients are dynamically programmable on the requirement. So that the reconfigurable constant coefficient multiplier architecture is needed for designing the image filter [1].

In this paper the designed Base- 2 common sub expression algorithm, reduces the complexity in Bsae-2 common sub expressions form. For designing the image filter with low complexity and power optimized scenarios the reconfigurable constant coefficient multiplier is needed.

Gaussian Mask with image convolution is important algorithm for image denoising of linera filter design. Gaussian filter $(G)$ is defined for the following below equation.

$$
G(x, y)=\frac{1}{2 \pi \sigma^{2}} e^{-\left(x^{2}+y^{2}\right) / 2 \sigma^{2}}
$$

Where $\mathrm{G}$ is the kernel mask, $\mathrm{x}$, $\mathrm{y}$ are the image coordinates and $\sigma$ is the Gaussian parameter. The deviation of $\sigma$ is large, the image smoothing is higher.for this design $\sigma=1$.

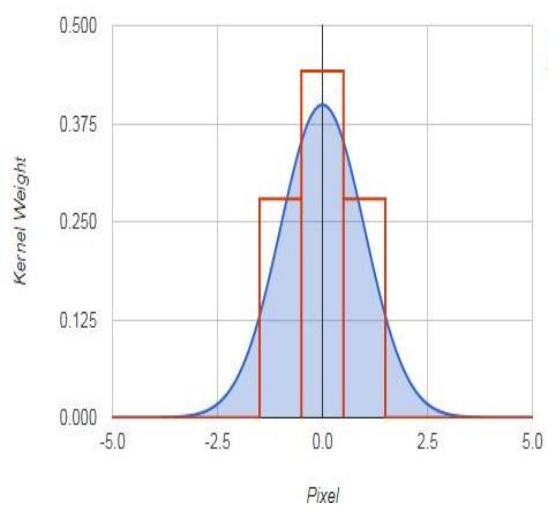

Fig.2. Image 2D-convolution using Gaussian mask with $\sigma=1$

Table-I: A $3 \times 3$ Test Image

\begin{tabular}{|c|c|c|}
\hline P1 & P2 & P3 \\
\hline P4 & P5 & P6 \\
\hline P7 & P8 & P9 \\
\hline
\end{tabular}

The image pixels are stored in $3 \times 3$ matrix is shown in table 1 .

Each image pixel is read from the memory and scanned in a sequential order for the atapath performing filtering operation with designed kernel.

Table-II: Gaussian 3×3 Mask

\begin{tabular}{|l|l|l|}
\hline 0.077847 & 0.123317 & 0.077847 \\
\hline 0.123317 & 0.195346 & 0.123317 \\
\hline 0.077847 & 0.123317 & 0.077847 \\
\hline
\end{tabular}

\section{PROPOSED ALGORITHM AND HARDWARE DESIGN}

Constant multiplication operation between the input image samples and the Gaussian kernel coefficients, for which the word length of the coefficients is 8-bit and it can be written as,

$\left(\frac{x i m}{2}\right) \cdot a++\left(\frac{x i m}{4}\right) \cdot a b+\left(\frac{x i m}{8}\right) \cdot a 5+\left(\frac{x i m}{16}\right) \cdot a++\left(\frac{x i m}{32}\right) \cdot a 3+\left(\frac{x i n}{64}\right) \cdot a 2+\left(\frac{x i m}{128}\right) \cdot a 1+\left(\frac{x i m}{256}\right) \cdot a 0$

Table-III: Constant coefficients design

\begin{tabular}{|c|c|c|c|c|c|c|c|c|}
\hline Coefficients & \multicolumn{7}{|c|}{ Binary weights } \\
\hline $\mathrm{a}$ & $\frac{x i n}{2} a 7$ & $\frac{x i n}{4} a 6$ & $\frac{x i n}{8} a 5$ & $\frac{x i n}{16} a 4$ & $\frac{x i n}{32} a 3$ & $\frac{x i n}{64} a 2$ & $\frac{x i n}{128} a 1$ & $\frac{x i n}{256} a 0$ \\
\hline $\mathrm{b}$ & $\frac{x i n}{2} b 7$ & $\frac{x i n}{4} b 6$ & $\frac{x i n}{8} b 5$ & $\frac{x i n}{16} b 4$ & $\frac{x i n}{32} b 3$ & $\frac{x i n}{64} b 2$ & $\frac{x i n}{128} b 1$ & $\frac{x i n}{256} b 0$ \\
\hline $\mathrm{c}$ & $\frac{x i n}{2} c 7$ & $\frac{x i n}{4} c 6$ & $\frac{x i n}{8} c 5$ & $\frac{x i n}{16} c 4$ & $\frac{x i n}{32} c 3$ & $\frac{x i n}{64} c 2$ & $\frac{x i n}{128} c 1$ & $\frac{x i n}{256} c 0$ \\
\hline $\mathrm{d}$ & $\frac{x i n}{2} d 7$ & $\frac{x i n}{4} b 6$ & $\frac{x i n}{8} d 5$ & $\frac{x i n}{16} d 4$ & $\frac{x i n}{32} d 3$ & $\frac{x i n}{64} d 2$ & $\frac{x i n}{128} d 1$ & $\frac{x i n}{256} d 0$ \\
\hline $\mathrm{e}$ & $\frac{x i n}{2} e 7$ & $\frac{x i n}{4} b 6$ & $\frac{x i n}{8} e 5$ & $\frac{x i n}{16} e 4$ & $\frac{x i n}{32} e 3$ & $\frac{x i n}{64} e 2$ & $\frac{x i n}{128} e 1$ & $\frac{x i n}{256} e 0$ \\
\hline $\mathrm{f}$ & $\frac{x i n}{2} f 7$ & $\frac{x i n}{4} b 6$ & $\frac{x i n}{8} f 5$ & $\frac{x i n}{16} f 4$ & $\frac{x i n}{32} f 3$ & $\frac{x i n}{64} f 2$ & $\frac{x i n}{128} f 1$ & $\frac{x i n}{256} f 0$ \\
\hline $\mathrm{g}$ & $\frac{x i n}{2} g 7$ & $\frac{x i n}{4} b 6$ & $\frac{x i n}{8} g 5$ & $\frac{x i n}{16} g 4$ & $\frac{x i n}{32} g 3$ & $\frac{x i n}{64} g 2$ & $\frac{x i n}{128} g 1$ & $\frac{x i n}{256} g 0$ \\
\hline $\mathrm{h}$ & $\frac{x i n}{2} h 7$ & $\frac{x i n}{4} b 6$ & $\frac{x i n}{8} h 5$ & $\frac{x i n}{16} h 4$ & $\frac{x i n}{32} h 3$ & $\frac{x i n}{64} h 2$ & $\frac{x i n}{128} h 1$ & $\frac{x i n}{256} h 0$ \\
\hline $\mathrm{i}$ & $\frac{x i n}{2} i 7$ & $\frac{x i n}{4} b 6$ & $\frac{x i n}{8} i 5$ & $\frac{x i n}{16} i 4$ & $\frac{x i n}{32} i 3$ & $\frac{x i n}{64} i 2$ & $\frac{x i n}{128} i 1$ & $\frac{x i n}{256} i 0$ \\
\hline
\end{tabular}

2-bit Base-2 common sub expression algorithm (2BCSE): The designed 2-bit base-2 common subexpression algorithm generated partial products from each case given as

$$
\frac{x i n}{2} a 7+\frac{x i n}{4} a 6+\frac{x i n}{8} a 5+\frac{x i n}{16} a 4+\frac{x i n}{32} a 3+\frac{x i n}{64} a 2+\frac{x i n}{128} a 1+\frac{x i n}{256} a 0 \quad \ldots-\cdots(1)
$$

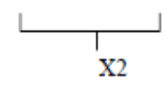

$$
\begin{aligned}
& \text { a. } x 1=y 1 \\
& x 2+\frac{1}{4}\left\{\frac{x 1}{2}+\frac{x 1}{4}\right\}+\frac{1}{16}\left\{\frac{x 1}{2}+\frac{x 1}{4}\right\}+\frac{1}{64}\left\{\frac{x 1}{2}+\frac{x 1}{4}\right\} \\
& x 2+\frac{x 2}{4}+\frac{x 2}{16}+\frac{x 2}{64}-
\end{aligned}
$$

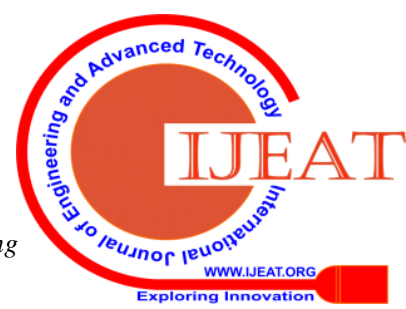




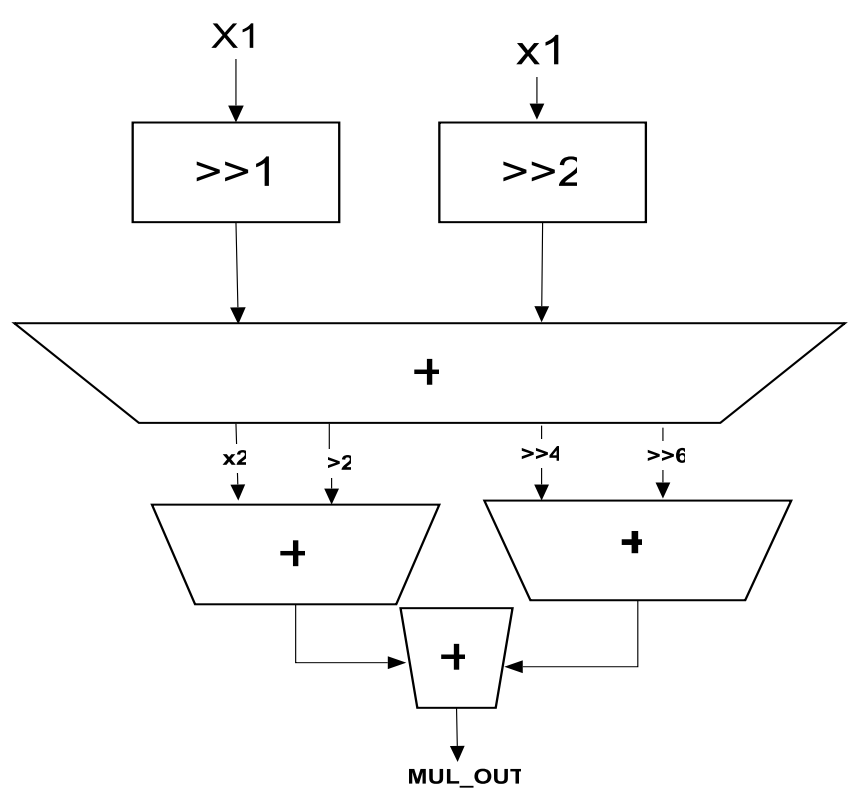

Fig.3: 2- bit Base-2common subexpression algorithm (2BCSE).

\section{3-bit Base-2 common sub expression algorithm (3BCSE):}

The designed 3-bit base-2 common sub expression algorithm used partial products from each case is given as

$$
\frac{x i n}{2} a 7+\frac{x i n}{4} a 6+\frac{x i n}{8} a 5+\frac{x i n}{16} a 4+\frac{x i n}{32} a 3+\frac{x i n}{64} a 2+\frac{x i n}{128} a 1+\frac{x i n}{256} a 0
$$

$$
\begin{aligned}
& \underbrace{\frac{x i n}{2} a 7+\frac{x i n}{4} a 6+\frac{x i n}{8} a 5+\frac{x i n}{16} a 4+\frac{x i n}{32} a 3+\frac{x i n}{64} a 2+\frac{x i n}{128} a 1+\frac{x i n}{256} a 0}_{\mathrm{x} 2} \\
& \begin{array}{l}
x 2+\frac{1}{4}\left\{\frac{x 1}{2}+\frac{x 1}{4}\right\} \\
x 2
\end{array}+\frac{1}{16}\left\{\frac{x 1}{2}+\frac{x 1}{4}\right\}+\frac{1}{64}\left\{\frac{x 1}{2}+\frac{x 1}{4}\right\} \\
& \underbrace{x 2+\frac{x 2}{4}+\frac{x 2}{16}+\frac{x 2}{64}}_{\mathrm{x} 3}
\end{aligned}
$$$$
x 3+\frac{1}{16}\left\{x 2+\frac{x 2}{4}\right\}
$$$$
\underbrace{x^{3}+\frac{x 3}{16}}
$$

The number of adders are reduced further by one. compaerd to 2-bit BCSEalgorithm. This Algorithm requires totally 15 Shift operations and 4 addition operations for performing 8-bit multiplication operation.

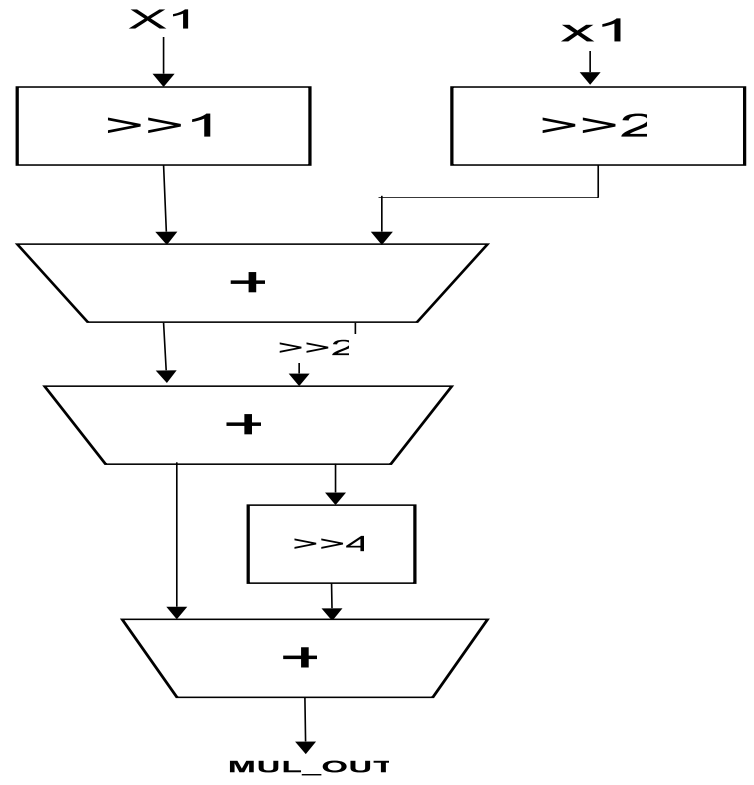

Fig.4: 3-bit Base-2 common sub expression algorithm (3BCSE).

The input image is converted into .coe file. The Mat lab image tool used for generating .coe file. An image pixel controller is designed using Finite State Machine (FSM)

based image controller to control the access of stored image binary values in the Block RAM.

In Block RAM the memory controller is controlling the flow

\begin{tabular}{|c|c|c|c|}
\hline Used Element & $\begin{array}{l}\text { Existed } \\
\text { (FBCSE) }\end{array}$ & \multicolumn{2}{|c|}{ Proposed } \\
\hline & & 3-bit BCSE & 2-bit BCSE \\
\hline Adders & $\begin{array}{c}4 \text { (8-bit, } 3 \text { (9-bit) } \\
\text { Total =9*7=63. }\end{array}$ & $\begin{array}{c}\text { 1(8-bit), } 2(9-\text { bit }) \\
\text { Total }=9 * 3=27\end{array}$ & $\begin{array}{c}1 \text { (8-bit), } \\
2 \text { (9bit), } 1(10 \text {-bit) } \\
\text { Total }=9 * 4=36\end{array}$ \\
\hline $\begin{array}{c}\text { Multiplexers/AND } \\
\text { Gates }\end{array}$ & $\begin{array}{l}8(8 \text { input AND } \\
\text { logic circuits }) \\
\text { Total }=9 * 8=72 .\end{array}$ & $\begin{array}{c}3 \text { Mux's(2:1 Mux's) } \\
\text { Total =9*3=27. }\end{array}$ & $\begin{array}{c}2 \text { Mux's( } 4: 1 \text { and } \\
2: 1) \\
\text { Total }=9 * 2=18 .\end{array}$ \\
\hline Propagation Delay & $\begin{array}{c}8(19 \mathrm{~ns})+7(3.25 \mathrm{~ns}) \\
=166.75 \mathrm{~ns}\end{array}$ & $\begin{array}{c}3(3.25 \mathrm{~ns})+3(3.01 \mathrm{~ns}) \\
=18.04 \mathrm{~ns}\end{array}$ & $\begin{array}{c}4(3.25 n s)+2(3.58 n s) \\
=20.16\end{array}$ \\
\hline
\end{tabular}
of image pixels and kernel pixels using pixel and mask controller blocks.

\section{RESULTS AND DISCUSSION}

Table- IV: Complexity analysis of Designed algorithm.

The complexity analysis is performed with existed and designed 2-bit ,3-bit BCSE algorithms. The proposed algorithm used 27 addrers and 27 multiplexers for 3-bit BCSE and 36 addres and 18 multiplexers are for 2-bit BCSE . 
Table -V: Comparison Results of the Designed Image filter on FPGA.

\begin{tabular}{|c|c|c|c|c|c|}
\hline & Target Device & LUT'S & Flip flops & SLICES & $\begin{array}{l}\text { Number of } \\
\text { buffectrls }\end{array}$ \\
\hline Proposed & \multirow{5}{*}{$\begin{array}{l}\text { Xilinx vitex-5 } \\
\text { Xc5VST-250 }\end{array}$} & & & & \\
\hline 2-bit BCSE & & 435 & 167 & 5 & 18 \\
\hline 3-bit BCSE & & 412 & 118 & 4 & 15 \\
\hline Existed & & & & & \\
\hline FBCSE & & 758 & 112 & 6 & 23 \\
\hline
\end{tabular}

The table[5] shows that designed algorithm used the less ${ }^{2}$ resources compared to the existed Fixed binary common subexpression (FBCSE).

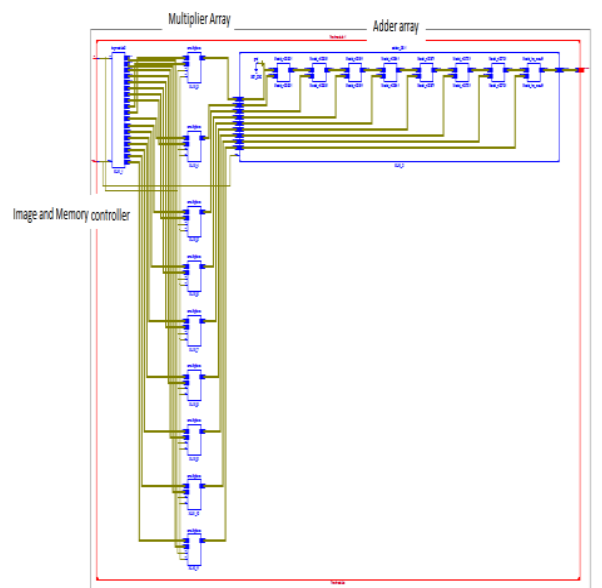

Fig.5: RTL schematic of designed Image filter.

The RTL schematic used 9 multipliers and 8 summing elements with image controller.

Table-VI: Comparative results of Power consumption.

\begin{tabular}{|c|c|c|c|}
\hline Parameter & \multicolumn{2}{|c|}{ Proposed } & Existed \\
\hline \multirow{3}{*}{ Power (mw) } & $\begin{array}{c}\text { 2-bit BCSE } \\
\text { MULTIPLIER }\end{array}$ & $\begin{array}{c}\text { 3-BIT BCSE } \\
\text { MULTIPLIER }\end{array}$ & $\begin{array}{c}\text { FBCSE(Fixed base-2 common } \\
\text { sub expression) }\end{array}$ \\
\cline { 2 - 4 } & $165 \mathrm{mw} @ 200 \mathrm{MHZ}$ & 129mw@ 200MHZ & 227mw@200MHZ \\
\hline $\begin{array}{c}\text { Critical path } \\
\text { delay(ns) }\end{array}$ & 12.294 & 11.3 & 15.6 \\
\hline
\end{tabular}

Table-VII: FPGA Resources profile of 2D Image filter using Proposed Algorithm.

\begin{tabular}{|c|c|c|c|}
\hline \multicolumn{4}{|c|}{ Device Utilization Summary } \\
\hline Logic Utilization & Used & Available & Utilization \\
\hline Number of bonded IOBs & 74 & 960 & $7 \%$ \\
\hline Number of buffctrls & 16 & 32 & $3 \%$ \\
\hline Number of DSP48Es & - & 192 & $0 \%$ \\
\hline
\end{tabular}

The designed BCSE algorithm based multiplier is tested in the image convolution. The $3 \times 3$ pixel matrix used 9 multipliers and which are connected in parallel. So that the speed is increased.

\section{CONCLUSION}

3 bit BCSE and 2-bit BCSE is implemented on Reconfigurable architecture using FPGA with less complexity. The number of resources utilized in the designed multiplier is reduced. The designed multiplier tested in the FIR based Gaussian filter and verified the number of resources are reduced to 90 . The $2 \mathrm{D}$ convolution process for $3 \times 3$ image required clock cycles are 3 approximately. and power consumption of designed image $2 \mathrm{~d}$-convolver is takes 100mw for 3-bit BCSE and 165me for 2 bit BCSE.

\section{REFERENCES}

1. Darak.S, Gopiand.S and Lai.S.,"Low-complexity reconfigurable fast filter bank for multi-standard wireless receivers," IEEE Trans.(VLSI) System, vol. 22, no.5, pp.1202-1206, May 2014.

2. Zhu, Zheng.Q and Xiong.D,“3D tubular structure extraction using kernel-based super ellipsoid model with Gaussian process regression”. IEEE Visual Communications and Image Processing (VCIP),vol.22,no.2.pp.1-6,2012.

3. Foia and Borachi,"An isotropically foveated non local image denoising filter". $20^{\text {th }}$ IEEE International Conference on Image Processing (ICIP), Vol.2, pp. 464-468, 2013.

4. Letourneau, E.Verhaeghe and J.Reader, "various weighted least squares algorithm based tracer distribution, count level, iterations and post-smoothing on PET quantification", IEEE Nuclear Science Symposium and Medical Imaging Conference(IEEE), vol.2,pp.2351-2353, 2012

5. Vega-Rodriguez,Gomez-Pulido, “An optimized reconfigurable hardware architecture for implementing image con volution ". Proceedings of the 2004,World Automation Congress, 16, pp.131-136, 2004

6. C. Y. Yao, H.Chen, "A Novel common subexpression elimination method for fixed-point FIR filters," IEEE Trans. Circuits Syst. , Reg. Papers, vol.51, no. 11, pp. 2215-2221, Nov. 2005

7. M. Aktan and A. Yurdakul, "An algorithm for the design of low-power hardware-efficient FIR filters,” IEEE Trans. Circuits Syst.I, Reg. Papers, vol. 55, pp. 1536-1545, Jul. 2008.

8. C. Y. Yao and Y. H. Ho, "Designing hardware-efficient fixed-point FIR filters,'IEEE Trans. Circuits and Systems, vol.61, no.1, pp. 202-212, 2014.

9. Dempster A.G an. Macloed M.D., "Use of minimum-adder multiplier blocks in FIR digital filters," IEEE Trans. Circuits Syst. II, Analog Digit. Signal Process. vol. 42, no. 9, pp. 569-577, Sep. 1995.

10. http://www.xilinx.com/itp/xilinx10/books/manuals.pdf

\section{AUTHORS PROFILE}

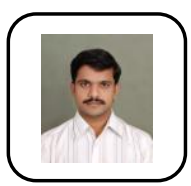

N. Samba Murthy obtained his B.TECH from JNTU Hyderabad and M.TECH from Gudlavalleru Engineering college, Gudlavalleru and presently pursuing Ph.D from JNTUK, Kakinada in the research area of VLSI architecture Design. Areas of interest are Microcontrollers, Embedded System Design, Microprocessors and VLSI architecture Design. He is a member of IE. Presently working as Assistant Professor in ECE Department, Gudlavalleru Engineering College, Gudlavalleru.

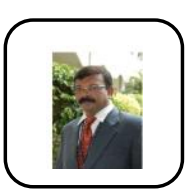

Dr. M. Kamaraju obtained his BE and M.E from the Andhra University and Ph.D from JNTUH, Hyderabad, in the area of Low Power VLSI Design. His areas of interest are microprocessors, microcontrollers, digital system design, embedded system design and low power VLSI design. He published 74 technical papers in national and international journals and conferences. He reviewed number of papers for international journal and conferences. He is a Fellow of the IETE, IE and a member of IEEE. He is presently working as a Professor and a mentor of the ECE Department, Gudlavalleru Engineering College, Gudlavalleru, India. He is the past Chairman of the IETE and IE State Wide Centre, Andhra Pradesh. He is also an executive counselor member of the JNTUK, Kakinada. 